\title{
Developing interprofessional education online: An ecological systems theory analysis
}

\begin{abstract}
This article relates the findings of a discourse analysis of an online asynchronous interprofessional learning initiative involving two UK universities. The impact of the initiative is traced over three intensive periods of online interaction, each of several weeks duration occurring over a three-year period, through an analysis of a random sample of discussion forum threads. The corpus of rich data drawn from the forums is interpreted using ecological systems theory, which highlights the complexity of interaction of individual, social and cultural elements. Ecological systems theory adopts a life course approach to understand how development occurs through processes of progressively more complex reciprocal interaction between people and their environment. This lens provides a novel approach for analysis and interpretation of findings with respect to the impact of pre-registration interprofessional education and the interaction between the individual and their social and cultural contexts as they progress through three/four years of their programmes. Development is mapped over time (the chronosystem) to highlight the complexity of interaction across microsystems (individual), mesosystems (curriculum and institutional/care settings), exosystems (community/wider local context) and macrosystems (national context and culture). This article illustrates the intricacies of students' interprofessional development over time and the interactive effects of social ecological components in terms of professional knowledge and understanding, wider appreciation of health and social care culture and identity work. The implications for contemporary preregistration interprofessional education and the usefulness and applicability of ecological systems theory for future research and development are considered.
\end{abstract}

Key terms: Ecological systems theory; discourse; professional identity; interprofessional education; online learning.

\section{Introduction}

The development of professionalism is a key feature in the socialization processes of health and social care professionals (Baker, Egan-Lee, Martimianakis \& Reeves, 2011; Khalili, Orchard, Spence-Laschinger \& Farah, 2013), whilst the demand for 
students to develop both a professional identity and the capacity for interprofessional working is rising (Hood, Cant, Leech, Baulch \& Gilbee, 2013). The development of appropriate professionalism continues to exercise the medical profession (Monrouxe 2010) and has challenged other health and social care professions not least when situated in the context of interprofessional education initiatives (McNair, 2005). Approaches to curriculum design for professional education programmes focused on knowledge and skill acquisition have been deemed limited in scope in the context of contemporary challenges (Dall'Alba, 2009). Considering that 'professional practice incorporates not only our knowing and how we act but also who we are as professionals,' Dall'Alba suggests that knowledge and skills are gained in order to contribute to a process of 'becoming' but they can only be developed in relation to social practices, of which the interprofessional milieu is a natural element.

The process of 'becoming' and how students' identities are shaped, formed and continually flex in response to demands and ubiquitous change, is a fascinating and challenging proposition for education providers responsible for preparing students for practice. Implicit in this statement is the suggestion that identity is a social construct that is not 'given' but is a fluid on-going process. Nor is it unitary; professional and interprofessional identities interact with one another, by virtue of 'perforate boundaries' (Clouder, Davies, Sams \& McFarland, 2012) that allow either identity to be dominant at any time depending on context (Burford, 2012). However, a sense of identity developed through the professional socialization process invokes 'in-group' (with their own profession) and 'out-group' (with other professions) dynamics (Brewer, 1979), which allows self-categorization or the ability to distinguish 'who one is not from a sense of who one is' (Burford, 2012). Having categorized 'them' and 'us,' individuals begin an identification process with the chosen group (their profession), which leads to a shared identity and a need to compare favourably with other 'out' groups. However, research suggests that individuals who identify strongly with their in-group, and have developed a sense of their own professional identity, have greater confidence in sharing this with others and are also positive about other groups therefore exhibit a readiness to engage in IPL (Hind et al., 2003; Richardson et al., 2010). This suggests that by enabling students to develop trust and mutual respect for each other, both within their professional group and across aligned professional groups, from the start of professional socialization, their ability to work 
interprofessionally is enhanced as their confidence grows. The 'out group' becomes a group with expertise and new knowledge, with whom shared issues regarding patient care can be solved more easily and more satisfactorily for the person at the centre of care.

There is agreement that identity development is dependent on everyday choices and a myriad of variables encountered (Vgotsky, 1978). The corollary of this stance is to consider interprofessional development in its widest sense spanning individual, institutional, local, and national influences over time. Incorporating these layers of complexity requires an expansive theoretical framework to enhance understanding that can inform successful implementation of interprofessional education initiatives.

\section{Background}

Reeves et al. (2007) have highlighted the importance of underpinning educational activities and practice with theories to enhance the evidence base. These authors completed a scoping review of organizational and educational theories relevant to interprofessional practice and education, that suggests the need to explore both the social and experiential learning characteristic of IPE. Further investigation of the potential contribution of organizational or systems theories has been suggested (e.g. Suter et al., 2013).

One of the most ambitious frameworks produced that does adopt a systems theory type approach, reflecting a need for a understanding of the complexity of interaction across levels, is the 'interprofessional education for collaborative patient-centred practice framework' (IECPCP) (D’Armour \& Oandasan, 2005). This framework aims to link determinants and processes of collaboration at micro, meso and macro levels to connect interprofessional education to interprofessional practice. Multifaceted and multiple levels of interactions are also recognized in a report identifying three discourses of professionalism (Hodges et al., 2011). They are identified as:

- An individual characteristic, trait, behaviour, or cognitive process

- An interpersonal process or effect

- A societal/institutional phenomenon: a socially constructed way of acting or being, associated with power. 
Hodges et al. (2011) set out the discourses without privileging any, although each of the discourses in isolation seems inadequate to explain the complexity involved in 'becoming interprofessional'. Whilst individual and group/ team level theories are of interest, again system level theories seem to offer a more all-encompassing means of making sense of the effectiveness of IPE initiatives. Mele, Pels and Polese (2010) have defined a systems theory as: "a theoretical perspective that analyses a phenomenon seen as a whole and not as simply the sum of elementary parts. The focus is on the interactions and on the relationships between parts, in order to understand an entity's organization, functioning and outcomes' (p.126).

System theories have been widely used across the natural and social sciences. Examples informing contemporary interprofessional education, include the use of cultural-historical activity theory (CHAT) to explore interprofessional collaboration in teams (Bleakley, 2013) and the use of actor network theory (ANT) and complexity theory, as part of a 'theoretical toolbox' to inform the design of interprofessional education initiatives (Hall, Weaver \& Grassau, 2013). Whilst CHAT acknowledges that human interaction with the environment is always mediated by culture, tools and signs, ANT focuses on interaction, including inanimate objects as actors. Of particular appeal with regard to the investigation providing the focus for this article, was ecological systems theory (Bronfenbrenner, 1979), which to our knowledge has not been used previously to investigate interprofessional education. Ecological systems theory was chosen as it seemed to offer a hierarchical framework that reflected the layers of scope of influence over time.

\section{Ecological Systems Theory}

Bronfenbrenner's $(1979 ; 1986 ; 1995)$ ecological systems theory adopts a life course approach to understand how development occurs through processes of progressively more complex reciprocal interaction between people and their environment. It has been used to illuminate a range of diverse fields including higher education (Renn \& Arnold, 2003; Poch, 2005), although most extensively in child development. The individual is located within a whole ecosystem which shapes interactions and outcomes by constraining and facilitating in a myriad of ways. Bronfenbrenner identifies five levels of interaction or 'spheres of influence', proposing that these are nested. Shown in Figure 1, they include: micro, meso, exo, macro and chronosystems. 


\section{INSERT FIGURE 1 ABOUT HERE}

Applied to the current study, microsystem influences include individual factors, such as past and present roles, lived experiences, interactions and friends. Mesosystem influences are organizational or institutional factors that shape or structure the environment, for example, the curriculum and teaching and learning approaches used. Bronfenbrenner claimed that the richer the medium for communication in the mesosystem the more influential it is on the microsystem. Exosystem influences come from the wider local context, therefore include community level influences that can be physical or spatial, but not necessarily. For example, professional norms, standards and social networks have community level impact, whilst the enablers and constraints of specific care settings or workplaces are also influential. The influence of macrosystems is easily identified due to the magnitude of impact, although they are not necessarily explicit in that they are the not solely geographical or physical but emotional and ideological. For example, the impact of western and UK culture on attitudes and beliefs about the delivery of care or attitudes to gender and social class etc. Finally, the chronosystem is important in the context of the current research in that the focus is on how students develop over time during the course of their programme.

\section{Research Context}

The interprofessional education initiative, the Interprofessional Learning Pathway (IPLP), provided opportunity for interaction of health and social care students (dietetics, learning disability nursing, medicine, nursing, mental health nursing, midwifery, occupational therapy, operating department practitioners, paramedics, physiotherapy, rehabilitation engineers, social work and youth work) from two neighbouring universities. The IPLP married IPE at scale and the pedagogical aspiration to design for rich interprofessional interaction. With approximately 1,200 students participating at each level, online asynchronous interaction was adopted as the most effective means of overcoming logistical constraints. The virtual learning environment, 'Moodle', provided the specific social context for interaction through an online discussion forum. The second notable feature of the IPLP was its continuity throughout the entire three-year programme (or four-year programme for dietetics), taking the form of 4 weeks in year 1 (first semester), year 2 (second semester) and within the final year (second semester). This threefold exposure to IPE ran alongside 
profession-specific curricula and naturally drew on the practice-based learning elements. This was achieved by the development of learning objects which engaged students in discussions surrounding authentic patient stories, co-created with the patients and practice colleagues. Stories were divided up into discreet segments which were released over time to build a complete account of patient experiences and supported by tailor-made activities which encouraged students to explore both the personal and professional experiences situated within the learning object. Our primary aim was to look for interprofessional development over the three/four years evident in online asynchronous discussions to see if we could identify any change in how students saw themselves as professionals, the status of their professional knowledge and understanding, and their wider appreciation of health and social care culture. We appreciated that any such change could not be attributed to the IPE curriculum per se but that the discursive approach to IPLP provided a vehicle through which to identify outcomes at discrete points within their development.

\section{Methods}

The research aim was to investigate interaction between students within the IPLP to evaluate its appropriateness to meet its aims of promoting interprofessional development. Online discussion forums provide a social context for individuals to use written language to develop and share their ideas in an asynchronous situation that allows time for reflection and the creation of shared versions of a social world. The research approach adopted assumed a subjectivist - constructivist orientation which involved a qualitative discourse analysis of coherent speech, contained within the online discussion forums as written text.

Discourse analysis is concerned with 'the meaning that events and experiences hold for social actors' (Wetherell, Taylor \& Yates, 2001, p.1). The organization of talk as joint activity and as communication, sheds light on the process of sense-making, construction of identity and the emergence of collective and individual mind, culture and social relations (Wetherell et al., 2001). In adopting a discourse approach and a social ecological lens, we accepted that all communication is a performance with recognizable effects and is context driven. In other words, the discourse of interprofessional learning (IPL) was socially and contextually constructed, and in 
addition performance driven. As such, students' comments are a proxy measure of their interprofessional development.

\section{Data collection}

Given the existence of 70+ groups at each level the corpus of available data was vast, necessitating sampling only a small proportion of the total. Purposive sampling was used to select four online discussion forums for analysis by a colleague external to the research team, at year 1, 2 and final year. This gave a total of 12 groups with 180 students participating by posting within these groups. The number of groups and associated number of students provided a manageable, but also a comprehensive set of data for each year. Each online discussion group contained up to 15 health and social care professional students drawn from approximately 1,200 students participating in the IPLP at each level. Disparities in numbers of students from different professional groups, meant that every group make-up differed. Individual posts in each discussion forum within the sample, ranged from 568 to 678 individual posts. The posts sit within discussion threads, which represent student conversations. As the written text itself is the data, data collection simply meant downloading the chosen groups' posts and anonymizing them by removing students' names and key identifiers, and replacing with their profession, gender, and a number. In downloading the posts, associated etivities were embedded within the data to give both clarity and context to the data examined. This led to large sets of data with approximately 100 single sides of hard copy for each group.

\section{Data Analysis}

The data presented within each group, across each year, was scrutinized by one member of the team. Our focus was on larger units rather than isolated words and sentences; we concentrated on examining patterns and commonalities across texts, considering what was being said but also what the interaction accomplished within the forums, taking each year in turn. A thematic analysis of data (Braun and Clarke, 2006) was informed by the ecological systems theory lens (Bronfenbrenner, 1979) which sensitized the team to the potential layers of developmental interaction across the systems. Initial codes were shared with the team and consensus was achieved on clustering codes to systematically build a picture of recurrent themes that emerged at 
year 1, 2 and 3 levels. These themes were discussed and refined within research team meetings to create a picture of professional progression using the ecological systems theory lens (Bronfenbrenner 1979) to inform our understanding. The model worked well and illuminated the developmental nature of both professional understanding and knowledge.

\section{Ethical Considerations}

Ethical approval was gained for this study from Coventry University Research Ethics Committee and accepted by Warwick Medical School. Due to difficulties with gaining written consent from such a large number of students, anyone not wishing to have their posts included in the corpus was encouraged to claim exemption by emailing the module lead. This group was then flagged and not accessed as part of the research study. The students were reminded about the research at the start of each discussion prior to any postings, but a reminder stayed on Moodle through the life of the group, and students could remove their consent as the discussion progressed.

\section{Findings}

Online comments that represent the discourses present in discussion forums illustrate the interaction between microsystem, mesosystem, exosystem and macrosystem influences at work within the context of the IPLP. The emerging themes that were evident in each year of the course and contributed to our understanding of how professional identity, professional knowledge and understanding and a wider appreciation of the culture developed across the life span of the course are examined year by year.

\section{Year One: Positionality and stereotypical lay perspectives}

Online conversations in year 1 discussion forums were typified by a 'getting to know one another' phase that was followed by discussion around set activities. A mix of 'lay' language and the tentative expression of newly acquired knowledge characterized early exchanges that at times revealed imprecision and errors of understanding and interpretation. For example, a male OT student referred to: 
"the 'welfare visitor' [who] would be able to advise any other health professionals working with the family' and a female medical student suggested 'a councillor [sic] would be the person to see regarding 'issues at home'."

However, conversely, the extensive life experience that students bring to their programme, through personal or family and friends' exposure to the health and care system, showed some to be well informed. Where opinion was based on individual life experiences this led in some cases to a lack of understanding of personal and professional boundaries, a potentially idealistic stance and unrealistic ideas for interventions. For example, a female social work student made an impassioned response to a scenario involving asylum seekers saying,

"I really think that the community needs to be educated about foreigners my experience has been that racism attacks people because they are different."

As online dialogue developed, unguarded opinion emerged on occasions, prompting challenge or rebuke, illustrating how online discussion provides opportunity for professional boundary testing in a safe context. For example, a female adult nursing student challenged another's comment by saying 'are you implying ... your statement appears to be judgmental', whilst a male medical student expressed his reservations about another comment saying,

"I'm uncomfortable with saying that the parents' religion will have a negative effect on the child's wellbeing."

The lay perspective was evident in the superficial knowledge structures patent in responses such as that made by a female learning disabilities nurse who suggested,

'the scenario shows bad practice ... everything social services should not be: unhelpful, not understanding and quite rude really'.

However, there was also evidence of well-informed perspectives. For example, in week one of the Year 1 IPLP, a male medical student uses typical medical language to express his views on the scenario saying, 
'malnutrition will also result in generally poorer health including depressed immune system functioning.'

A male social work student's response to the same scenario provides a contrasting view, privileging a social and holistic view. He suggests:

"I would be concerned initially with the lack of money to buy food. The overall feeling of isolation felt by the mother should not be overlooked ... if there is no money for basic provisions the family will be facing debt and possible homelessness. This situation can lead to a variety of health conditions."

The data suggested that the language used by professional groups differed. Medical students spoke in ways consistent with the language of the medical model, for instance, taking issues of 'malnutrition' and a 'depressed immune system' from the scenario. Contrast this with the social work student's concern with the social and psychological issues relating to malnutrition which stem from 'lack of money to buy food' and the 'feeling of isolation' reflecting a bio-psychosocial perspective. This conversation occurred 5 weeks after first starting their professional programmes and already there is a marked difference in the way an individual's circumstances were viewed in relation to students' world views and knowledge of the macrosystem.

In response to the activity that asked students to consider what role a member of their professional group would have in a certain situation students imagined themselves in the situation. A male OT student reflected,

'I have been thinking more as [name] the OT, rather than [name] the student. I have been developing my thinking so that I understand each scenario as if I was treating these people.'

A female adult nurse suggested,

'as a nurse in the community, I would have to ensure that she understood a balanced and healthy diet for the baby', while a female medical student noted, 'as a medic, I would only be able to ensure their health remains at its optimum. I would provide support to the family and ensure I arrange any counselling if it is necessary'. 
These comments illustrate that, as early as in the first semester, students are trying on the mantle of their chosen professions and 'acting' out the part (Goffman, 1978). Microsystem influences tend to dominate in the early weeks of the IPLP. Students adopt a largely egocentric and individualistic ' $I$ ' perspective as integral to the emergence of their professional persona, which is possibly more clearly defined because it emerges in an interprofessional context.

\section{Year 2: Transitioning from 'I' to 'we'}

By the next IPLP period, during the second semester of Year 2, there was a marked difference in student interaction. Students were quicker to settle into discussing issues and more confident in their assertions; a change that we attribute primarily to them having been exposed to the realities of practice experiences. They had learned at firsthand something of the subtleties of working with patients and clients, and alongside members of their own profession, and others; the exosystem influences has begun to influence attitudes and perceptions. A wealth of experience, good and bad, had broadened their vision to see their own aspirations in the context of their professions. The discourse was less focused on individualistic demonstrations of knowledge and jostling to be seen to be most knowledgeable, to one of speaking from within the professional in-group and sharing perspectives. With increased confidence in the parameters of their profession, they adopt the term 'we' as opposed to 'I' more commonly in conversation and can advocate for their professions. For example, a female adult nurse sums up a scenario saying

'in regards to the scenario where there are problems related to alcohol consumption, a nurse's primary role is to provide care in a nonjudgmental way.'

Students' dialogue had changed in character so that rather than speculating they could offer sound and knowledgeable advice to their peers, often backing this up with evidence. It is clear that by this point they are able to reflect their professional voice in a more informed manner and with a professional confidence that was absent in year 1. They could represent their professional view and were starting to develop the confidence to offer this to other professionals and experience how that was received. It was also evident that they were also more likely to challenge and critique practice. For example, a female social work student reflects: 
'I had a look at the Cochrane review 2010 as I was concerned re your comments that the 'Stages of Change' model is thought to be ineffective, especially as the British Association of Social Work has quoted it in their 2010 publication 'Social workers pocket guide on Alcohol and Drugs'. After reading the article it prompted me a little more and I found an online article ..., which gave a good account .... It made me think how readily I accepted the model because it was published by a professional body without questioning it'.

Firmly established within their chosen group, there was recognition that there were others outside of their group that they were working alongside, that they could cooperatively work with to both share and challenge relevant information and practice. Therefore, stereotypical views of professional groups were replaced with more realistic understanding of the roles and responsibilities of their professional colleagues, as the following excerpt illustrates:

'I have attended safeguarding and child protection meetings where MDT's are working together to make considerable changes for the better to improve the effected individual, their family and their carer's supported needs'(Female Mental Health Student)

Students' confidence to express themselves within their interprofessional group had grown and enabled them to feel safe to consider their own limitations as well as what it might be like to work in a collaborative manner (within the mesosystem). They had a developing grasp of the care systems and the individuals who populate it, in which they had been working (exosystem) and were beginning to appreciate the implications and impact of external factors, such as 'duty of care' and accountability. They showed that they were starting to test out what they bring to the profession but equally what they needed to learn and develop to become an acceptable member of the profession. However, there was only limited engagement with the exosystem. The expectation that once out in practice students would identify with and be absorbed into the interprofessional activity present around them was not found, probably because they had yet to be sure of themselves; they were concentrating on immersing themselves in their own culture and discovering the special nature of their professional groups.

Final year: Interprofessional allegiances and getting on with the job in hand 
Discourse evident in the interprofessional online groups in Year 3 portrayed students' recognition of the necessity of being part of a whole system approach to care, the expertise of the different professionals within this system and the centrality of service-users and their carers; they became small cogs in a larger organization working together to deal with the issues they encountered. At this level, students can be seen to move between all systems drawing on a multitude of diverse influences. The conversations were less about 'who we are' and the role they played, and more about 'how we work.' For example,

'I have been working on a neurorehab ward and have found that informal discussions between professionals are very useful ... I find that this is something not documented but vital information to know, and if you have this information you are more likely to understand your patient and build up a rapport' (Female Physio Student).

By the final year professionalism was at the forefront of the students' minds. The online discussions were characterized by critiques of practice that, although made with respect and sensitivity to their inteprofessional colleagues, nonetheless, demonstrated a capacity and willingness to challenge the status quo of practice settings. Issues of cultural conformity were discussed and debated. Students were keen to explore the cultural norms that they were exposed to, seemingly able to cross professional boundaries with honest discussion. For instance, reflecting on an experience in practice, a female OT student addresses the issues without apportioning blame:

As none of the nursing staff on duty that day had any idea this patient was being discharged until a few hours before, they also didn't have the opportunity to prepare her emotionally and mentally for the move. This showed a lack of communication, disorganization and overall a huge negative impact on this patient.

The online scenarios proved to stimulate equally insightful discussion and served to demonstrate students' integrating a wide range of knowledge drawn from microsystems (observations of individual practitioners, personal values etc), the exosystem (professional standards, policies etc) and macrosystem (primarily the fallibility of care systems). For example: 
I think that all three HPCs had the patient's best interests at heart but unfortunately the hospital's policies and procedures were not up to date in accordance with evidence-base practice (Female OT student).

Of course, another solution would be to have more social workers with reduced caseloads, but we don't always live in an ideal world. It should be something that government should aim for (Female student midwife).

It was not until the final year that the exosystem was fully understood and embraced by the student group. Repeated episodes of experience in practice interspersed with relevant theoretical exposure, enabled students to move forward to greater understanding of their interprofessional identity by consolidating their grasp of their own practice and online interprofessional experiences, to create bridges to cogent multi and interprofessional understanding. The shift seemed focused on the students' ability to understand the importance of a wider context for service-user care. This understanding also drew on a greater appreciation of the impact of time on the other systems. For example, a female OT student says, 'this occurred many years ago and I believe that services have changed in this area' and a female medical student adds,

'I don't believe it will be as big an issue in the future of our professions, as it was maybe 20 years ago.'

\section{Discussion}

The aim of the research was to investigate interaction between students within the IPLP to evaluate its appropriateness to meet its aims of promoting interprofessional development. Online discourse occurring during three episodes of IPE exposure has been analyzed using ecological systems theory as a lens to understand the changes that were observed, and to discover the building blocks that may help to facilitate interprofessional development. The discourse across the three years demonstrates how this development occurs through processes of progressively more complex reciprocal interaction between people and their social and environmental context. Figure 2 illustrates the interaction between microsystem, mesosystem, exosystem and macrosystem influences at work within the context of the IPP. 
The three interrelated themes to emerge from the interaction of these influences were the development of professional identity, professional knowledge and understanding, and a wider appreciation of health and social care culture. These themes provide an understanding of the key aspects of the IPLP that were central to the development of a student's interprofessional identity across the life span of the course. These findings confirm Dall'Alba's (2009) suggestion that the acquisition of knowledge and skills occurs in the context of social practices, which are crucial to the emergence of students as developing professionals.

A sense of continuum and self-growth across the three or four years of study was apparent. Early in year 1 of the programme, self-categorization was evident as students clearly wished to be recognized as individuals who knew what they were doing and recognized for who they were. Identification processes progressed to students speaking knowledgeably as part of their uniprofessional group (year 2). 'Acting' the part (Goffman, 1978) of an emerging professional seemed to be a precursor to considering themselves to be a member of a larger team with service users as the focus of the care (year 3). In essence students moved from concentrating almost exclusively on themselves, to developing an outward-looking focus, and a professional identity that was flexible enough to be subordinated to an interprofessional identity, where context required (Burford, 2012).

Year 1 discourse was characterized by a certain amount of naivety and lay knowledge, although some students were well informed. It is tempting to predict that microsystem level influences would be most dominant early in the programme yet given that individual attitudes and behaviours are borne out of socialization processes, macrosystem and exosystem influences undoubtedly played an implicit part in shaping students' responses. There was more tangible evidence of awareness of the influence of the environment and the players that students anticipated working with, within Year 1, as they shared a speculative understanding of the exosystem from their sometimes, stereotypical perceptions of the professions. The ecological systems theory framework (Bronfenbrenner 1986) allowed us to clearly identify the range of systems that impacted on students even at this early stage in their courses, which resonates with and sheds light on the complexities associated with becoming professional (Hodges et al., 2011). 
Year one discourse illustrates the status of early knowledge structures, perhaps typical of the different professions. Many are based on propositional knowledge and some early practical know-how that will develop to become procedural and tacit knowledge during the professionalization process (Eraut, 1994). However, the evidence also indicated the positionality of students in terms of their self-categorization and identification with their chosen professions and their wish to be 'seen' to be part of the in-group of their profession, distinct within the interprofessional group. This represented an important stage of identity development emphasized by the occurrence of uniprofessional 'jostling' to be seen as 'authentic,' and perhaps most knowledgeable by their own group.

The greatest influence at this stage in the programme probably comes from mesosystem influences of the curriculum, and teaching and learning approaches adopted. The online interaction in the IPLP may be a significant aspect of the mesosystem in that Bronfenbrenner (1986) claimed that the richer the medium for communication in the mesosystem the more influential it is on the microsystem. Certainly, our findings suggest that the online nature of the IPLP allows students to try out their ideas and even their professional personas in a relatively safe context.

During Year 2 of the programme, students experience the practice environment and the enactment of their professional personas. It is here that exosystem influences are brought to bear in the realities of practice, which have a powerful impact on students' sense-making. It is over-ambitious to expect that students will move beyond profession-specific depth of understanding at this level. However, we see that by Year 3 they have come to see their profession in its wider context. Only then do they adopt a service orientation and appreciation of what it might mean to collaborate as part of an interprofessional team. Our findings support the suggestion that students who have developed a sense of their own professional identity have greater confidence in interacting with others from different professional groups (Hind et al. 2003). By this stage students are aware of flaws in the system and can critique practice on many levels. Whether or not this would lead to action to provoke change is unknown. 'Top down effects' (Oetzel, Ting-Toomey \& Rinderle, 2006) or structural factors, such as community and institutional influences, and professional and regulatory systems, are powerful in shaping an individual's agency, and therefore their responses. However, 
at least students show that they are aware of the realities or practice before they are fully immersed in it and lose sight of some of their ideals.

The inclusion of the chronosystem in ecological systems theory fits neatly with the focus on tracing interprofessional development over students' three/four-year programmes. Students were seen to mature as they progressed through their programmes through exposure to the many influences of the other systems. The impact of time is felt through the way in which programmes are constructed and how much time is devoted to different activities. For instance, time spent on interprofessional activities might be perceived to be time lost from more valuable profession-specific activities unless supported by powerful messages about the importance of looking beyond profession- specific goals, which were present in the IPLP partner institutions. The iterative nature of the IPLP provided insight into students' appreciation of the pace of change in the health and social care landscape as they came to understand macro and exosystem influences. They demonstrated awareness of policies, service delivery models, organizational structures, professional regulatory frameworks, employment options and advances in professional practice that make the future difficult to predict. The implications for keeping abreast of change were clearly recognized by students, who displayed a readiness to adapt to the demands of contemporary practice.

Wenger (1998, p. 229) notes that 'learning cannot be designed; it can only be designed for' and suggests that curricula design reflects how learning is understood. Therefore, if we believe that interprofessional identity is socially constructed, investigating interaction between students can provide insight into the appropriateness of the IPE initiative to meet its aims. The IPLP activities and instructions whilst important serve only as a vehicle to inspire sharing ideas that draw on a wide range of influences across all of the system levels. The occurrence of IPE in the curriculum has been hotly debated, arguments variously suggesting it can be too early or not early enough (Dalrymple, Hollins \& Smith, 2013). Based on findings, our suggestion is to gradually and incrementally introduce IPE to provide exposure across several years and integrating it as an iterative and interactive proposition. The ecological systems framework has highlighted the importance of giving students a means of demonstrating their developing interprofessional identities over time, both for their 
own purposes, and also the teaching team if they need convincing of the place of IPE in the curriculum. All IPE interventions will be open to students drawing on a wide range of influences to construct their understanding of interprofessional practice, and the findings presented are particular to the IPLP. Features such as its asynchronous online nature, its iterative structure, and its emphasis on reflection, mean that it is unique to the instituions in which it sits. Nevertheless, although not generalizable, findings confirm the link between carefully designed, closely monitored and regularly evaluated IPE and interprofessional development, offering ideas that are transferrable to other programmes.

Ecological systems theory has provided a useful framework from which to consider interprofessional development in the context of a pre-registration IPE initiative and is recommended as a useful addition to the theories within the 'theoretical toolbox' advocated by Hall et al. (2013). It provides a means of mapping potential influences on students and tapping into these to create menaingful experiences for those designing interprofessional education opportunities. Introducing students to systems thinking early in their programmes would potentially shift conceptions of the unrivalled value of uniprofessional propositional knowledge and practical know-how, which they tend to privilege, to recognizing other types, such as tacit knowledge, in the professionalization process (Eraut, 1994). Given that new professionals will continue to develop, taking on new roles, responsibilities and challenges that force a re-examination of their professional personas, personal skills, values, capabilities and knowledge, ecological systems theory would also help to explain how development progresses throughout professional careers. Sargeant (2009) argues that IPE in a continuing education context requires a new way of thinking that reflects the socially constructed nature of knowledge in practice. Further research might focus on the relative influence of micro, meso, exo and macrosystems over time as individuals move and adapt but also at the impact that individuals have on the systems that they enter.

\section{Concluding comments}

Exploring the IPLP through the layers of ecological systems theory has enabled us to consider the impact of engaging students in bursts of online interprofessional activity, 
interspersed throughout their programmes. We are encouraged to find that our aims of promoting interprofessional understanding and development appear to be being met. The scheduled activities provide a vehicle for students to build their professional understanding and knowledge, appreciation of profession specific and interprofessional culture, and associated identities. Our findings support the understanding that interprofessionalism is fundamentally shaped by a complex interplay of a wide range of factors that can be understood more fully within an ecological systems theory framework.

\section{Declaration of interest}

The authors report no conflicts of interest. The authors alone are solely responsible for the content and writing of this article. The research was not funded.

\section{References}

Baker, L., Egan-Lee, E., Martimianakis, M.M. \& Reeves, S. (2011). Relationships of power: implications for interprofessional education. Journal of Interprofessional Care, 25, 98-104. doi.org/10.3109/13561820.2010.505350.

Bleakley, A. (2013). Working in "teams" in an era of "liquid" healthcare: What is the use of theory? Journal of Interprofessional Care, 27, 18-26. doi.org/10.3109/13561820.2012.699479.

Bluteau, P. \& Jackson, A. (2009). An elearning model of interprofessional education. In P. Bluteau \& A. Jackson (Eds.) Interprofessional Education; making it happen, (pp107-121). London. Palgrave.

Braun, V. \& Clarke, V. (2006). Using thematic analysis in psychology. Qualitative Research in Psychology, 3 (2), 77-101.doi.org/10.1191/1478088706qp063oa.

Brewer, M. B. (1979). In-Group Bias in the Minimal Intergroup Situation: A Cognitive-Motivational Analysis. Psychological Bulletin, 86(2), 307-324.

Bronfenbrenner, U. (1979). The ecology of human development. Cambridge, MA: Harvard University Press.

Brofenbrenner, U. (1986). Ecology of the family as a context for human development: Research perspectives. Developmental Psychology, 22, 723-742.

Bronfenbrenner, U. (1995). Developmental ecology through space and time: a future perspective. In P. Moen, G. H. Elder \& K. Luscher (Eds.) Examining lives in context: perspectives on the ecology of human development, (pp. 619-647). Washington DC, American Psychological Association. 
Burford, B. (2012). Group processes in medical education: learning from social identity theory. Medical Education, 46, 143-152. doi:10.1111/j.13652923.2011.04099.x

Clouder, D.L., Davies, B., Sams, M. \& McFarland, L. (2012). Understanding where you are coming from; Discovering an [inter]professional identity through becoming a peer facilitator Journal of Interprofessional Care. 26(6), 459-464. doi: 10.3109/13561820.2012.706335.

Dall'Alba, G. (2009). Learning Professional Ways of Being: Ambiguities of becoming. Educational Philosophy and Theory, 31(1), 34-45. DOI: 10.1111/j.14695812.2008.00475.x

D'Armour, D. \& Oandasan, I. (2005). Interprofessionality as the field of interprofessional practice and interprofessional education: An emerging concept. Journal of Interprofessional Care. 19(1), 8-20.

Dalrymple, L., Hollins, M. \& Smith, W. (2013) Improving understanding of teaching strategies perceived by interprofessional learning lecturers to enhance students' formulation of multidisciplinary roles: An exploratory qualitative study. Journal of Research in Interprofessional Practice and Education, 3(1), 78-91.

Eraut, M. (1994). Developing professional knowledge and competence. London, Routledge Falmer.

Goffman, E. (1959). The Presentation of Self in Everyday Life. London, Penguin.

Hall, P., Weaver, L. \& Grassau, P. A. (2013). Theories, relationships and interprofessionalism: Learning to weave. Journal of Interprofessional Care, 27(1), 73-80. doi.org/10.3109/13561820.2012.736889.

Hean, S., Craddock, D. \& Hammick, M (2012). Theoretical insights into interprofessional education: AMEE Guide No. 62. Medical Teacher, 34: e78e101. doi: 10.3109/0142159X.2012.650740.

Hind, M., Norman, I., Cooper, S., Gill, E., Hilton, R., Judd, P. \& Jones, S. (2003). Interprofessional Perceptions of Health Care Students. Journal of Interprofessional Care, 17(1), 21-34.

Hodges, B.D., Ginsberg, S., Cruess, R., Cruess, S., Delport, R., Hafferty, F., (...) Wade, W. (2011). Assessment of professionalism: Recommendations from the Ottawa 2010 Conference. Medical Teacher, 33: 354-363. doi: 10.3109/0142159X.2011.577300.

McNair, R. P. (2005). The case for educating health care students in professionalism as a core content of interprofessional education. Medical Education 39(5), 456-464.

Hood, K., Cant, R., Leech, M., Baulch, J. \& Gilbee, A. (2013). Trying on the professional self: nursing students' perceptions of learning about roles, 
identity and teamwork in an interprofessional clinical placement. Applied Nursing Research, 27(2), 109-114.

Khalili, H, Orchard, C., Spence-Laschinger, H.K. \& Farah, R. (2013). An interprofessional socialization framework for developing an interprofessional identity among health professional students. Journal of Interprofessional Care, 27(6), 448-453. doi.org/10.3109/13561820.2013.804042.

Mele, C., Pels, J. and Polese, F. (2010). A Brief Review of Systems Theories and Their Managerial Applications. Service Science, 2(1/2), 126 - 135.

Monrouxe, L.V. (2010). Identity, identification and medical education: why should we care? Medical Education, 44(1), 40-49. doi:10.1111/j.13652923.2009.03440.x

Oetzel, J. G., Ting-Toomey, S. \& Rinderle, S. (2006). Conflict communication in contexts: A social ecological perspective. In J. G. Oetzel \& S. Ting-Toomey (Eds.) The Sage Handbook of conflict communication. Thousand Oaks, CA, Sage.

Poch, S. (2005). Higher education in a box. International Journal of Education Management. 19(3), 246-258.

Reeves, S. \& Hean, S. (2013). Why we need theory to help us better understand the nature of interprofessional education, practice and care. Journal of Interprofessional Care, 27(2), 103. doi.org/10.3109/13561820.2013.751293.

Reeves, S., Suter, E., Goldman, J., Martimianakis, T., Chatalalsingh, C., DeMatteo, D.J. (2007). A scoping review to identify organizational and education theories relevant for interprofessional practice and education. Canadian Interprofessional Health Collaborative Consortium.

Renn, K.A. \& Arnold, K.D. (2003). Reconceptualising peer culture on college student peer culture. The Journal of Higher Education. 74(3), 261-291.

Richardson, J., Letts, L., Childs, A. \& Semogas, D., Stavness, C. Smith, B. J., (...) Price, D. (2010). Development of a Community Scholar Program: An Interprofessional initiative. Journal of Physical Therapy Education, 24(1), 3743.

Sargeant, J. (2009). Theories to aid understanding and implementation of interprofessional education. Journal of Continuing Education in the Health Professions 29 (3), 178-184.

Suter, E., Goldman, J., Martimianakis, T., Chatalalsingh, C., DeMatteo, D.J. \& Reeves, S. (2013). The use of systems and organizational theories in the interprofessional field: Findings from a scoping review. Journal of Interprofessional Care, 27(1), 57-64. doi.org/10.3109/13561820.2012.739670.

Vygotsky, L. S. (1978). Mind in Society: The development of higher psychological processes. Cambridge, MA, Harvard University Press. 
Wenger, E. (1998). Communities of Practice: Learning, Meaning, and Identity. Cambridge, Cambridge University Press.

Wetherell, M., Taylor, S. \& Yates, S. J. (2001). Discourse Theory and Practice. London, Sage. 